\section{JOURNAL OF THE SOUTH AFRICAN} INSTITUTION OF CIVIL ENGINEERING ISSN 1021-2019

Vol 59 No 1, March 2017, Pages 2-10, Paper 1445

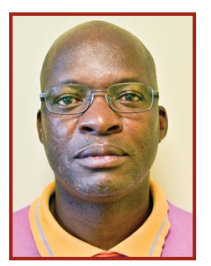

DR RAPHAEL MUKARO is a senior lecturer in the Department of Physics and Electronics at North-West University, Mafikeng Campus, where he has worked since 2015. From 2008 to 2014 he lectured Applied Physics at the University of KwaZulu-Natal (UKZNN), where he taught third-year modules (mainly Electronics, Signal Processing Techniques and Measurement Systems). He was also a senior lecturer in physics at Bindura University of Science from 1999 to 2007. He received his PhD degree from UKZN in 2014. His current research interests are in the digital, statistical and wavelet study of turbulence generated by laboratory-plunging water waves.

\section{Contact details:}

North West University

Mafikeng Campus

School of Mathematical and Physical Sciences

Department of Physics and Electronics

Private Bag X2046

Mmabatho

2735

South Africa

T: +27183892151

E: raphael.mukaro@nwu.ac.za

\title{
Vorticity filaments beneath regular turbulent flow
}

\author{
R Mukaro
}

Two-dimensional vorticity flow fields created in the wake of a plunging breaker were investigated for regular turbulent flow at a Reynolds number of 30000 . Velocity flow fields obtained from an earlier study that had employed digital particle image velocimetry, were analysed to determine vorticity shedding patterns and the interactions between the vorticity filaments as flow progressed. Central difference approximations were applied to the velocity fields to determine vorticity at each point in the field. Most of the strong instantaneous vorticity observed in the flow field was in the form of filaments. A hierarchy of filaments of different lengths were observed, with the longest being as long as the height of the wave used. During the early phases of the flow, instantaneous vorticity tended to organise into thin filaments of counter-rotating pairs. Eventually, the co-rotating vorticity filaments coalesced and ultimately merged in the turbulent flow as flow progressed, while counter-rotating vorticity filaments were cancelled by viscous dissipation. The results suggested that filaments travel more slowly than the wave velocity and drifted towards the bed as they became elongated, and the number of filaments remaining in the flow were observed to decrease as flow progressed. Whereas phaseresolved instantaneous vorticity results showed pairs of counter-rotating vorticity filaments near the crest, the phase-averaged vorticity description of flow fields showed a dominant primary positive vorticity filament around the shear boundary layer.

\section{INTRODUCTION}

Wave breaking is one of the most important problems for coastal engineers. The breaking process leads to either surging, spilling or plunging breaker types. A plunging breaker is the most violent breaker, initiated when a steepening wave crest curls over to form an overturning jet. Steepening of the wave naturally induces high curvature and consequently strong vorticity. The jet plunges into the water ahead to form a plunger vortex, and also produces a splash-up leading to strong turbulence in the flow. The generated turbulence is a collection of weakly correlated vortical motions, which, despite their intermittent and chaotic distribution over a wide range of space and time scales, actually consist of local characteristic eddy patterns that persist as they move around under the influences of their own and other eddies' vorticity fields (Hunt \& Vassilicos 2000). Energy in the wave is transferred from the average flow to vortex structures at different scales. Due to the non-linear interactions between different scales, cascade processes of energy are very complex (Lin et al 2002). Wave-breaking turbulence accounts for most of the sediment suspension through the introduction of eddies associated with breaking waves, with increasing levels of turbulence coming towards the boundary layer from the water surface.

Ocean and coastal engineers have been interested in wave loading generated by turbulence and extreme waves and their interaction with marine structures. Ryu et al (2007) investigated potential damage to coastal structures caused by significant impacts of breaking waves and associated overtopping greenwater. The formation of bed topography, which, as a result of a complicated interaction between flow and sediment particles along the bed, has also received considerable attention. Lajeunesse et al (2010) used a high-speed video-imaging system to record the trajectories of the moving particles over a flat bed, and observed that entrained particles exhibit intermittent motion, composed of the succession of periods of flight and rest. Keshavarzi and Ball (2011) suggested that the structure of turbulent flow over the ripples in the bottom of an open channel is important for understanding of sediment particle entrainment and its transport. Two important issues which they suggested need to be understood in sediment movement are the stochastic nature of instantaneous shear stresses over a ripple bed, and how it influences on sediment entrainment and transport. They employed an Acoustic Doppler Velocity Meter (Micro-ADV) with a sampling rate of $50 \mathrm{~Hz}$ to measure three-dimensional velocities, a charge-coupled device camera to record images of particle motion, and image-processing techniques to provide an accurate measurement of flow structure and particle entrainment from the bed. In 
a study of sediment entrainment from the bed, Williams (1990) and Nelson et al (1995) investigated and found a high correlation between the stream-wise velocity component and the sediment flux. Additionally, Nelson et al (1995) found that the transport rate tends to be higher when the vertical velocity and Reynolds momentum flux are angled towards the bed. They found the best correlation between the sediment flux and the stream-wise velocity component to occur with a lag of 0.1 second, and consequently they suggested that a measuring frequency of $10 \mathrm{~Hz}$ would give the best results in terms of time scales. Nielsen (1984) showed experimentally that the process of sediment suspension is convective rather than diffusive, and that it is dominated by such features as travelling vortices, jets and turbulent bursts. In addition, the effect of wave-breaking on the concentration profile in terms of turbulence associated with each breaker type was discussed. Experimental results near the bed showed that sediment concentration profiles are very similar, suggesting that the near-bed reference concentration is independent of the external turbulence (breaking waves) and is controlled mainly by the bottom boundary layer. Voulgaris and Collins (2000) observed that an efficient numerical model of time-averaged suspended sediment concentration should be related to the breaking characteristics of the waves. A commonly used parameter to define the onset of wavebreaking is the ratio of wave height to water depth. Galvin (1972) defined another simple wave-breaking parameter that is a function of breaking wave height $\left(H_{b}\right)$, beach slope $(\tan \beta)$ and wave period $(T)$ :

$\frac{H_{b}}{g T^{2} \tan \beta}=B_{b}$

For $B_{b}>0.068$ the breaking waves are spilling, for $0.003<B_{b}<0.068$ the waves are plunging, while surging breaking waves are present when $B_{b}<0.003$. Galvin's parameter has been used extensively in the study of the effect of the breaking-wave-induced vortices in sediment resuspension (Zhang \& Sunamura 1990; Zhang 1994). Zhang (1994) identified four types (A-D) of oblique rolling vortices. The conditions for the occurrence of the observed types of vortices were defined as a function of Galvin's breaker parameter (Equation (1)), and the breaking wave Reynolds number $R_{e}=H_{b} L_{b} / v T$ where $L_{b}$ and $v$ are the wavelength at the breaking position and the seawater kinematic viscosity, respectively. From laboratory experiments, Zhang (1994) found that oblique vortices whirl the bed material and lift the sediment up into suspension, acting as a

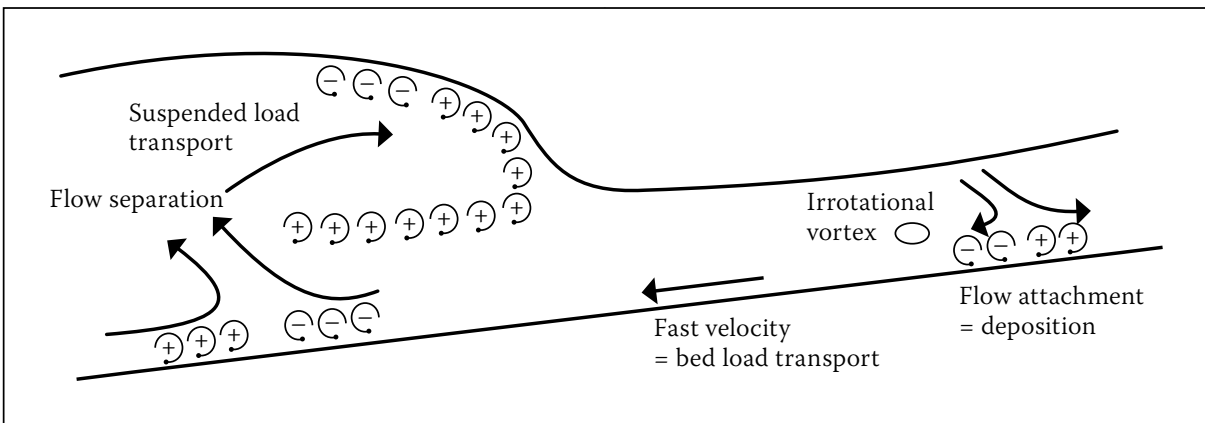

Figure 1 Schematic representation of the vorticity behaviour and corresponding flow structure in the surf and swash zones (Sou \& Yeh 2011)

tornado. They observed that types A and $B$ vortices last longer than types $C$ and $D$. Type A horizontal vortices act in a similar manner as the oblique vortices, but are capable of re-suspending more sediment. Type B horizontal vortices dig the bed material like a cultivator, so that a larger amount of sand is lifted up into suspension and is entrapped in the vortex. Types $C$ and $D$ do not reach the bottom and thus do not contribute to sediment re-suspension. Considering the duration that the vortex is in contact with the bed, the strength of the vortex and thus its capability to re-suspend sediment was found to be highest in the B-type horizontal vortex, decreasing in the A-type horizontal vortex and the oblique vortex, in that order.

The creation of vorticity due to dissipating or breaking waves is a basic fluid dynamics phenomenon that is central to many geophysical and engineering applications (Buhler 2000). As stated by Long and ÖzkanHaller (2009), vorticity motions in the near shore contribute to mixing and dissipation. The presence of vorticity induces shear at the water surface which changes the properties of the free surface waves when meeting an underlying current. Turbulent flows display intermittent pressure drops associated with vorticity concentrations, so pressure measurements can also be used to locate regions with high vorticity or dissipation in turbulent flows (Abry et al 1994). Bracket et al (1983) were the first to show, via simulations, that there is a direct relationship of the small pressure filaments with vorticity filaments. There have also been many experimental attempts to visualise coherent vorticity structures in boundary layers or in rotating flows, but only a few in the bulk of fairly homogeneous isotropic flows (Dernoncourt et al 1998). Sou and Yeh (2011) employed particle image velocimetry to investigate the fundamental characteristics of the flow structure in the vertical cross-shore plane as the wave evolves from the outer surf zone to the swash zone. They suggested that vorticity generated near the bed has a simple relation to the pressure gradient for boundary layer flows. The no-slip boundary condition, i.e. vanishing inertial force at the boundary, means that the phase-averaged momentum equation on a plane surface of a two-dimensional flow can be expressed (Sou \& Yeh 2011) as:

$\mu\left(\frac{\partial^{2} u}{\partial z^{2}}\right)=\mu \frac{\partial \omega_{y}}{\partial z}=\frac{\partial P^{\prime}}{\partial x}$

where $\mu$ is the dynamic viscosity and $P^{\prime}$ is the excess pressure at the bed surface, given by $P^{\prime}=P+\rho g z$, where $P$ is the atmospheric pressure, $\rho$ is the water density, $g$ is acceleration due to gravity and $z$ is the water depth. Equation (2) implies that the flux of vorticity from the bed is created by the pressure gradient. This indicates that when the pressure gradient in the $x$-direction is negative, the vorticity gradient in the $z$-direction $\partial \omega_{y} / \partial z$ is also negative, suggesting that the positive or clockwise $(\mathrm{CW})$ vorticity is generated at the bed during uprush (Sou \& Yeh, 2011). Figure 1 shows a schematic sketch by Sou \& Yeh (2011) that summarises the observed flow structure and flow characteristics of vorticity observed in the surf and swash zones, which show the resulting load deposition, transport and suspension. They observed that in the surf zone a thin layer of counter-clockwise (CCW) vorticity on top of the $\mathrm{CW}$ vorticity is generated along the water surface, due to strong induction of the CW vorticity by the collision of the uprush and downwash flows. The surface flow pattern can support the presence of CCW vorticity on the surface behind the surface roller. They also pointed out that the presence of CCW vorticity on the surface is necessary, based on the Stokes theorem. Sou \& Yeh (2011) also observed that flow separation occurs at the bore front, so the detached sediments from the bed by flow separation must remain suspended by the wave-breaking-generated turbulence and the shear-layer-generated turbulence. They also noted that the vorticity intensity at the shear layer decreases in magnitude in the offshore locations. In addition, suspended sediments should deposit on the bed with the flow attachment process as the 
wave-breaking-generated turbulence and the shear-layer-generated turbulence levels become relatively low at the flow reversal.

Dabiri and Gharib (1997) studied the vorticity generation within a spilling water wave using the particle image velocimetry technique. Douady et al (1991) studied turbulent Von Karman swirling flows using water seeded with air bubbles which displayed intermittent formation of filaments of bubbles, ascribed to vorticity concentrated on tube-like structures. Pressure measurements (Fauve et al 1993; Abry et al 1994) and pressure-velocity correlations (Cadot et al 1995) were used in the detection of vorticity filaments, and in the study of their dynamic and statistical properties. Dernoncourt et al (1998) used an ultrasound scattering technique that allowed measurement of the vorticity in the bulk of the flow. They used short-time Fourier transforming of the incoming sound frequency and filtering of the scattered pressure signal, allowing the detection of large vorticity concentrations on small spatial scales, but not the study of their detailed internal dynamics. Their essential findings were that the vorticity at small scales has a clear tendency to exhibit intense rare events that are associated with localised structures compatible with the visualisation of low-pressure structures in the same flow. Lien and Sanford (2000) observed that turbulence vorticity flux plays an important dynamic role in the turbulence boundary layer. They used similarity scaling of the turbulence vorticity flux to estimate the bed stress, and observed that turbulence vorticity flux was related to the divergence of turbulence momentum flux, which represents the turbulence force on the mean flow. They argued that understanding the spectral properties of the turbulence vorticity flux would help improve turbulence parameterisation schemes in numerical models.

Kevlahan and Farge (1997) stated that, in order to successfully model a twodimensional turbulent flow, it is essential to understand the role of vorticity filaments. They used the term 'filament' to refer exclusively to the thread-like structures observed in two-dimensional turbulence. Siggia (1981) was the first to show in a simulation that, in a turbulent flow, the regions in which vorticity had the largest modulus were in the shape of elongated filaments. Numerical studies by Jimenez et al (1993) have also shown that intense vorticity levels in turbulent flows are organised in Burger-type filaments. The same objects have been identified by locating local minima of pressure fields in numerical experiments (Belin et al 1996). Dritschel et al (1991) noted that a common feature of strongly non-linear, high Reynolds number, two-dimensional flows is the presence of thin filaments of vorticity, while Jimenez and Wray (1998) also observed that most of the strong vorticity in the flow field is in the form of filaments. According to Elhmaidi et al (2004), vorticity filaments are easily generated during vortex-vortex interactions (such as vortex merger), and when vortices are exposed to strong shearing. Kimmoun and Branger (2007) used calculations of the spatial derivatives of the velocity field measured through particle image velocimetry, in order to obtain relevant information on vorticity. Dritschel (1989) observed that, once large vortices are formed in a turbulent flow, likesigned vortices merge together in an inelastic process. They observed that, during the merger process, filamentation occurs, leading to the formation of thin strips of vorticity. While some filaments remain attached to the large coherent vortices, others are expelled during the merger and remain detached from the large vortices. In a related study on vorticity filaments in two-dimensional turbulence Kevlahan and Farge (1997) suggested that vorticity filaments form in twodimensional turbulence during the merger of coherent vortices, due to the strong shear forces that develop during the interaction. These forces pull some of the vorticity out into long, narrow strips of intense vorticity which are usually spiral in shape. Petitjeans (2003) also weighed in by pointing out that vorticity filaments are generated by roll-up of a fluid sheet around the vortex core, and that it is now well known that local stretching of vorticity in turbulent flows produces very intense vortices called vorticity filaments. Meunier et al (2005) studied the physics of vortex merging. They observed that at the beginning of the merging, two strong filaments of vorticity are ejected, and roll up around the final vortex due to the differential rotation, leading to an axisymmetric vortex at late stages. Just as in previous work by Kevlahan and Farge (1997), Elhmaidi et al (2004), Dritschel (1989), Jimenez et al (1993), Jimenez and Wray (1998), Meunier et al (2005) and Petitjeans (2003), narrow strips of intense vorticity are what is meant when referring to 'vorticity filaments' in this work. However, as pointed out by (Petitjeans 2003), the presence of vorticity does not imply the existence of a vortex, e.g. in shear boundary layers, where we may have vorticity but no vortices. Where a vortex is present, the term 'vorticity' describes its rate of rotation about some axis, locating and giving the vortex its strength or intensity.

Results presented herein are related to an earlier study in which experiments were performed in a laboratory wave flume to study the turbulence generated by $0.4 \mathrm{~Hz}$ breaking water waves (with a wave height of $12 \mathrm{~cm}$ ) as they propagate along a glass-walled laboratory flume and break on a 1:20 plane slope. Characterisation of the flow structure, description of the experimental setup and methods used have been previously reported in Mukaro and Govender (2011; 2013). Here it will suffice to just mention that the temporal and spatial evolution of instantaneous and phase-ensemble-averaged velocity flow fields induced were examined using flow visualisation and digital correlation image velocimetry. A computer-driven electronic measurement system was designed, developed and employed to capture images of breaking waves. An eight-bit, monochromatic, progressive-scan digital camera was connected to the computer and mounted on the side of the flume to capture images of these breaking waves. Through the use of a trigger pulse from the wave generator, the computer synchronises image acquisition by the camera at the instance the computer drives the strobe lights to illuminate the field of view. The wave cycle was subdivided into 20 overlapping phases or fields of view. For a particular phase, 100 sequential image pairs were captured and the information saved on a computer. Instantaneous velocities were later determined by measuring the combined displacement of polystyrene particles and air bubbles entrained in the flow. A digital correlation image velocimetry technique was employed to calculate the spatial cross-correlation of the grey-scale image data by means of computing the cross-power spectrum of the Fouriertransformed image samples. This yielded 100 instantaneous velocity flow fields for each phase. Velocity measurements obtained from this turbulent flow enabled the computation of instantaneous and mean flow vorticity. A single component of vorticity is obtained from velocity fields by measuring the rotation of particles seeded in a turbulent flow. The velocity data were differentiated to yield vorticity field data that characterises the wake of a plunging breaker. The spatial and temporal evolution of both instantaneous vorticity and vorticity of the mean flow are presented only for phases where turbulence is predominant.

\section{VORTICITY CALCULATION}

Vorticity of a flow field is defined as the curl of the velocity field. It is a vector field that gives a microscopic measure of the rotation at any point in the fluid, and plays a vital role in the dynamics of turbulent fluid flows. The vorticity vector plays an outstanding role in turbulence kinematics and dynamics of the flow (Wang 2012), and has been described as the principal quantity that defines the flow structure (Kim et al 1995; Wu et al 2006). If 
resolved temporally, the vorticity field can be much more useful in the study of flow phenomena than the velocity field (Raffel $e t$ al 1998). At any instant eddies are present in the flow, ranging in size from the largest geometric scales of the flow down to small scales where molecular diffusion dominates. These eddies are continuously evolving in time, and the superposition of their induced motions leads to the fluctuating time records normally observed. By calculating the vorticity fields from the velocity field it is possible to follow the motion of coherent structures in the flow.

For a three-dimensional fluid flow, vorticity can be calculated in Cartesian coordinates, from the partial derivatives of the velocity components as:

$$
\begin{aligned}
\vec{\omega}=\nabla \times \vec{u}= & \hat{x}\left(\frac{\partial w}{\partial y}-\frac{\partial v}{\partial z}\right)+\hat{y}\left(\frac{\partial u}{\partial z}-\frac{\partial w}{\partial x}\right) \\
& +\hat{z}\left(\frac{\partial v}{\partial x}-\frac{\partial u}{\partial y}\right)
\end{aligned}
$$

where $\hat{x}, \hat{y}$ and $\hat{z}$ are the unit basis vectors for the three-dimensional Euclidean space and $u$, $v$ and $w$ are velocities in the $\mathrm{x}, \mathrm{y}$, and $\mathrm{z}$ directions, respectively. The turbulent flow studied here has velocity fields confined only in the two-dimensional $\mathrm{x}-\mathrm{z}$ plane. This means that only one component of vorticity is present. This component, $\omega_{y}$, which is perpendicular to the flow and points in the orthogonal $\mathrm{y}$-direction is given (Sou \& Yeh 2011) as:

$\vec{\omega}_{y}=\hat{y}\left(\frac{\partial u}{\partial z}-\frac{\partial w}{\partial x}\right)$

Figure 2 shows data points on a computational grid of a velocity mesh used for calculating vorticity at a particular point $(i, j)$. The four yellow-shaded grid points were used to numerically estimate vorticity at this point.

Instantaneous vorticity at a given grid point $(i, j)$ was computed from derivatives of the instantaneous horizontal, $u_{i}$, and vertical, $w_{i}$, velocity components using second-order central difference approximation (Sou \& Yeh 2011; Lee \& Lee 2001) as :

$$
\begin{aligned}
\omega_{y}(i, j)= & \frac{u_{i}(i, j+1)-u_{i}(i, j-1)}{2 \Delta z} \\
& -\frac{w_{i}(i+1, j)-w_{i}(i-1, j)}{2 \Delta x}
\end{aligned}
$$

where $(i, j)$ represent the spatial grid point in the $\mathrm{x}$ - $\mathrm{z}$ plane, and $\Delta x$ and $\Delta z$ are the $\mathrm{x}$ and $z$-grid spacing, with values $4.1 \mathrm{~mm}$ and $5.0 \mathrm{~mm}$ respectively.

Vorticity of the mean flow is the transverse vorticity component calculated from the phase-ensemble averaged velocity components $\langle u\rangle$ and $\langle w\rangle$, given by Sou and Yeh (2011) as:

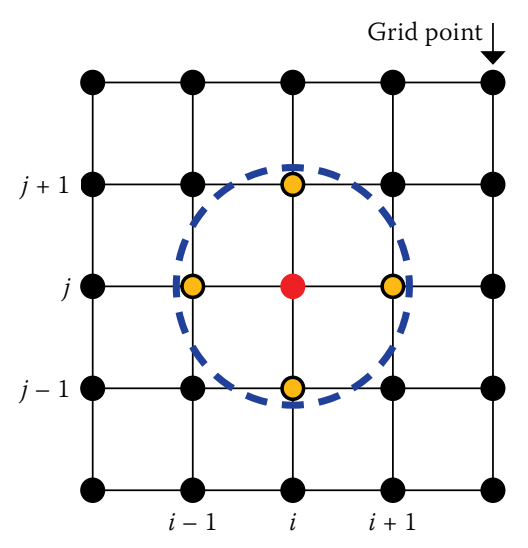

Figure 2 Velocity mesh grid used for calculating vorticity at point $(i, j)$ using second-order central difference approximation

$\left\langle\vec{\omega}_{y}\right\rangle=\hat{y}\left(\frac{\partial\langle u\rangle}{\partial z}-\frac{\partial\langle w\rangle}{\partial x}\right)$

This means that vorticity was estimated from the derivatives of the phase-ensembleaveraged velocity components, using the central difference method as:

$$
\begin{aligned}
\left\langle\omega_{y}(i, j)\right\rangle= & \frac{\langle u(i, j+1)\rangle-\langle u(i, j-1)\rangle}{2 \Delta z} \\
& -\frac{\langle w(i+1, j)\rangle-\langle w(i-1, j)\rangle}{2 \Delta x}
\end{aligned}
$$

\section{RESULTS AND DISCUSSIONS}

Spatially and temporally resolved velocity vector fields previously measured for a plunging breaker were used to investigate the phase evolution of the instantaneous and mean flow vorticity. For easier visualisation, the vorticity components are presented in the form of contour plots, and show evolution of the vorticity as flow progressed. In order to describe the two-dimensional configuration of the wake of this turbulent flow, instantaneous vorticity and vorticity of the mean flow were computed from the instantaneous velocity fields. It should, however, be emphasised that most flows of practical interest are three-dimensional, so vorticity behaves very differently in three dimensions, compared to two.

\section{Wave images}

Figure 3 shows a series of typical grey-scale images of a $0.4 \mathrm{~Hz}$ plunging breaking wave captured by a monochrome digital camera at six different phases at one station, where turbulence was observed to be predominant. These photographs were captured at one position and were intended to illustrate the qualitative features of the breaking waves that were analysed and presented in this paper. The temporal evolution of the breaking is shown in two space coordinates

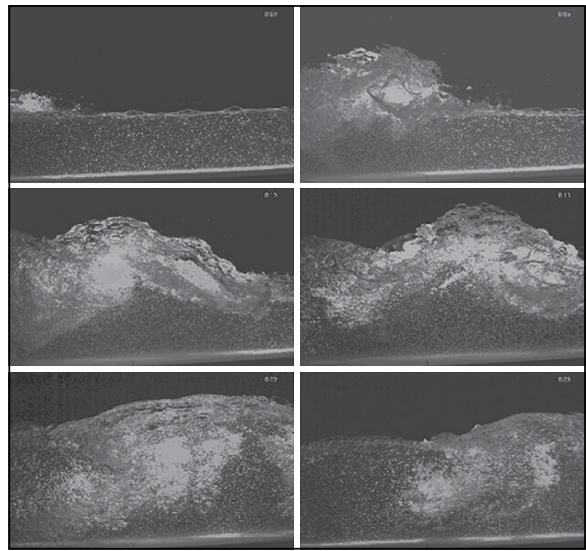

Figure 3 Typical grey-scale level images of the $0.4 \mathrm{~Hz}$ breaking wave showing six consecutive phases that include the crest; these images are for phases $\mathrm{t} / \mathrm{T}=$ $0.00,0.05,0.10,0.15,0.20$ and 0.25 ; the wave is propagating from left to right

in a series of photographs where the time separation between two consecutive frames is $0.125 \mathrm{~s}$. The magnitude of the intensity of each pixel in the images is represented by levels of grey from black to white. Images from the 20 wave phases are labelled relative to the wave period, with $\mathrm{t} / \mathrm{T}=0.00$ representing the first phase; 0.05 for the second, through to phase $\mathrm{t} / \mathrm{T}=0.95$ for the twentieth phase. These correspond to phases: $\mathrm{t} / \mathrm{T}=0.00,0.05,0.10,0.15,0.20$ and 0.25 . The waves propagate from left to right, and the images evolve from left to right, top to bottom, as shown in Figure 3. The photo for phase 0.00 (top left) shows an image of the breaking wave at the phase when the crest is just coming into the field of view of the camera. The last photo in Figure 3 shows the image at a later phase when the crest has just passed. White polystyrene beads that were used as tracer particles together with air bubbles to determine the velocity flow fields are clearly visible in the images. The white cap in the images shows aerated water. The breaking process is observed to create a high-speed roller that rides on the front face of the wave crest. The water mass in the roller is rotating about the horizontal axis that is parallel to the front face of the wave, creating a huge amount of turbulence and shear stress at the front face of the wave. Images at other phases show the trough part of the wave which is not as dynamic, so vorticity results only for the six phases presented.

\section{Instantaneous vorticity $\left(\omega_{y}\right)$}

Characteristics of vorticity structures in the turbulent flow, and their evolution as phase progresses, are presented in Figure 4, which shows the sequence of contour plots of instantaneous vorticity generated from the images. These reveal the evolution of the vorticity fields as flow progressed. These 
plots were obtained from the instantaneous velocity fields of the images presented in Figure 3. These six snapshots of the vorticity field are presented here to best illustrate spatio-temporal evolution of near-surface eddies during the passage of the wave crest. The colour bar shows the magnitude and direction of the vorticity. Colours are coded according to the intensity of the vorticity field from minimum vorticity (green) to the maxima (either red or blue). Positive vorticity indicates motion in clockwise rotation, and the direction is into the plane of the figure, while negative vorticity indicates anticlockwise rotation, with direction out of the plane of the figure. Positive vorticity has been conveniently taken to point in the positive y-direction. Red-filled contours indicate clockwise or positive vorticity, whereas the blue-filled contours represent counter-

clockwise or negative vorticity. The abscissa shows distance from the intersection of the still water line with the beach slope, and is taken as negative away from the shore. The still water line is at elevation $\mathrm{z}=0 \mathrm{~cm}$ on the contour plots.

The spatial variation of the instantaneous vorticity shows a series of strong counterrotating filaments observed behind the leading edge of the breaker. The presence of vorticity patches near the water surface shows that wave-breaking which occurs at the free surface is the major source of vorticity in the flow. The underlying physical mechanism for this vorticity near the surface was explained by Sou and Yeh (2011), who suggested that water on the front face must move faster than the propagation speed, hence the formation of a surface roller. On the other hand, the water behind the front face must move more slowly than the propagation speed, creating a divergence on the surface. The filaments are observed to diffuse to the bottom of the flume, reaching the bed after the crest has passed (phases 0.20 and 0.25). As pointed out by Longo (2008), deepening is quite fast immediately after breaking and is slower at subsequent phases. Pairs of counter-rotating filaments have peak vorticity of magnitude $100 \mathrm{~s}^{-1}$ which is observed to decrease as flow progresses. These patches have only been shown to exist, but the mechanism by which they are set up is not clear. However, they are understood to dissipate the remaining wave energy. It is the interactions between these adjacent counter-rotating filaments that produce a wake of complex vortex distribution behind the wave crest. These filaments, firstly observed at elevations above $z=-5 \mathrm{~cm}$ at phase 0.00 , originate from the free surface and obliquely propagate in the direction of the wave. Their presence is an indication of strong mixing taking
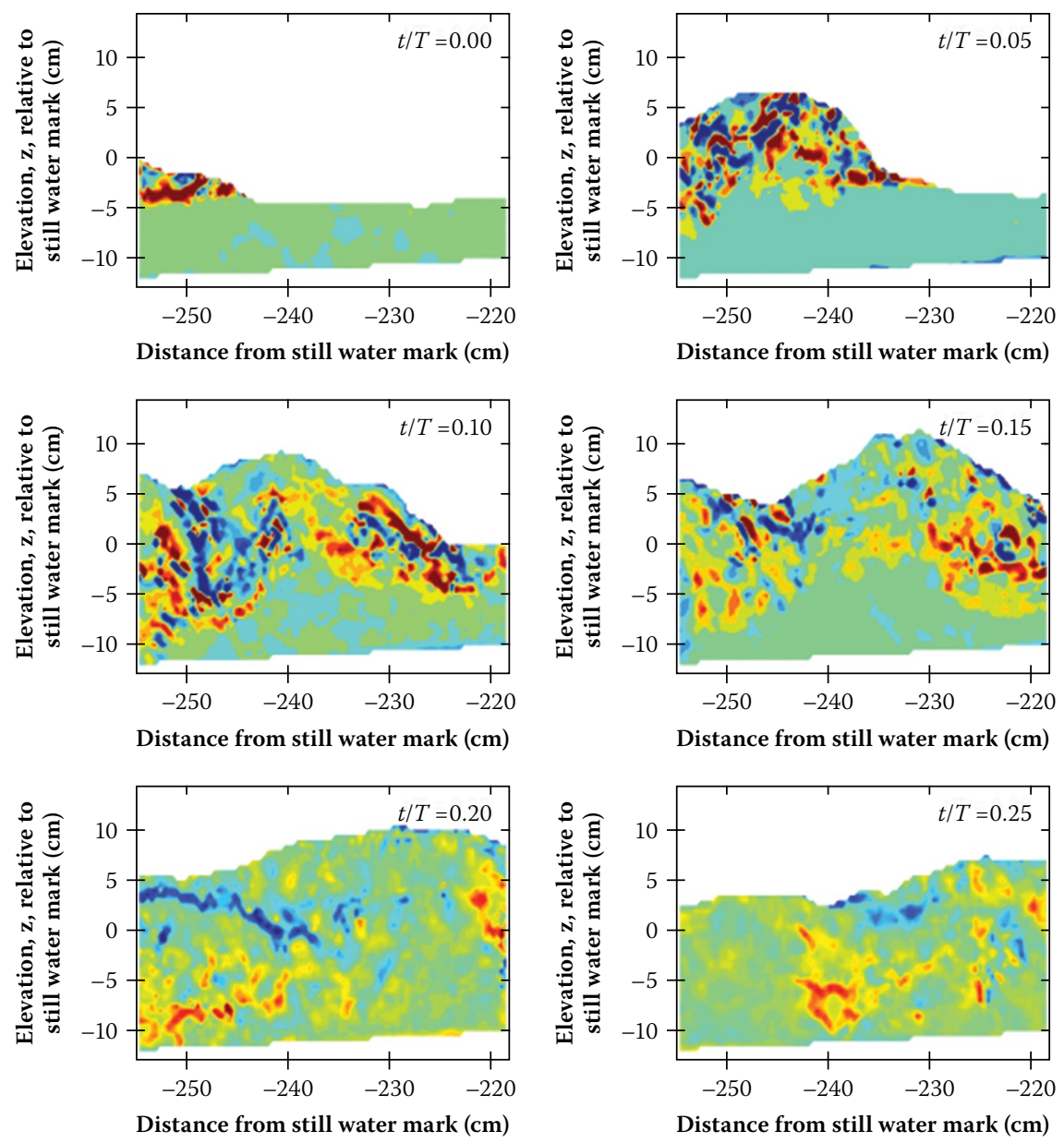

Figure 4 Contour plots showing the evolution of instantaneous vorticity filaments with phase, for breaking wave images shown in Figure 3; the still water line (SWL) is at $z=0 \mathrm{~cm}$

place there. Near the trough level there are occasional regions devoid of strong vorticity. Early phases also show large expanses of the fluid with nearly zero vorticity. Bakewell and Lumley (1967) and Aubry et al (1988) used the proper orthogonal decomposition in the near-wall region, and observed that a pair of counter-rotating stream-wise vortices contain the largest amount of energy. Ting (2006; 2008) used particle image velocimetry (PIV) to study instantaneous turbulent velocity fields associated with a broken solitary wave on a plane slope, and also observed that large eddies were composed of two counter-rotating vortices.

Just as parallel electric currents in the same direction attract one another because of their magnetic interaction, co-rotating filaments coalesce and ultimately merge in viscous flow as flow progresses, while counter-rotating vorticity filaments interfere with one another by viscous vorticity cancellation. Vorticity cancellation ultimately leaves predominantly weaker positive vorticity throughout most of the wake and negative vorticity confined to just below the saddle points, as seen in the last two panels of Figure 4. This cancellation is the key mechanism for the decay of two-dimensional vorticity distributions. Leweke et al (2016) illustrated this mechanism to show how, in a viscous flow, two identical co-rotating vortices ultimately merge, while counter-rotating ones cancel. Coalescing of co-rotating vorticity filaments is evidenced by the eventual formation of a positive vorticity filament ring observed to be centred around $x=-240 \mathrm{~cm}$ in the last panel of Figure 4. Negative vorticity (anticlockwise) filaments are observed to disappear as a result of mixing, leaving the fluid volume filled mainly with positive, clockwise filaments. The filaments deepen and diffuse towards the bottom, as shown in phases $0.10-0.25$. Saddle points near the crest are characterised by strong filaments of obliquely descending positive and negative vorticity below them.

Characteristics of instantaneous vorticity structures in the flow, such as the vorticity filament width (core diameter in three-dimensional vorticity), length and translational speed of the filaments between 


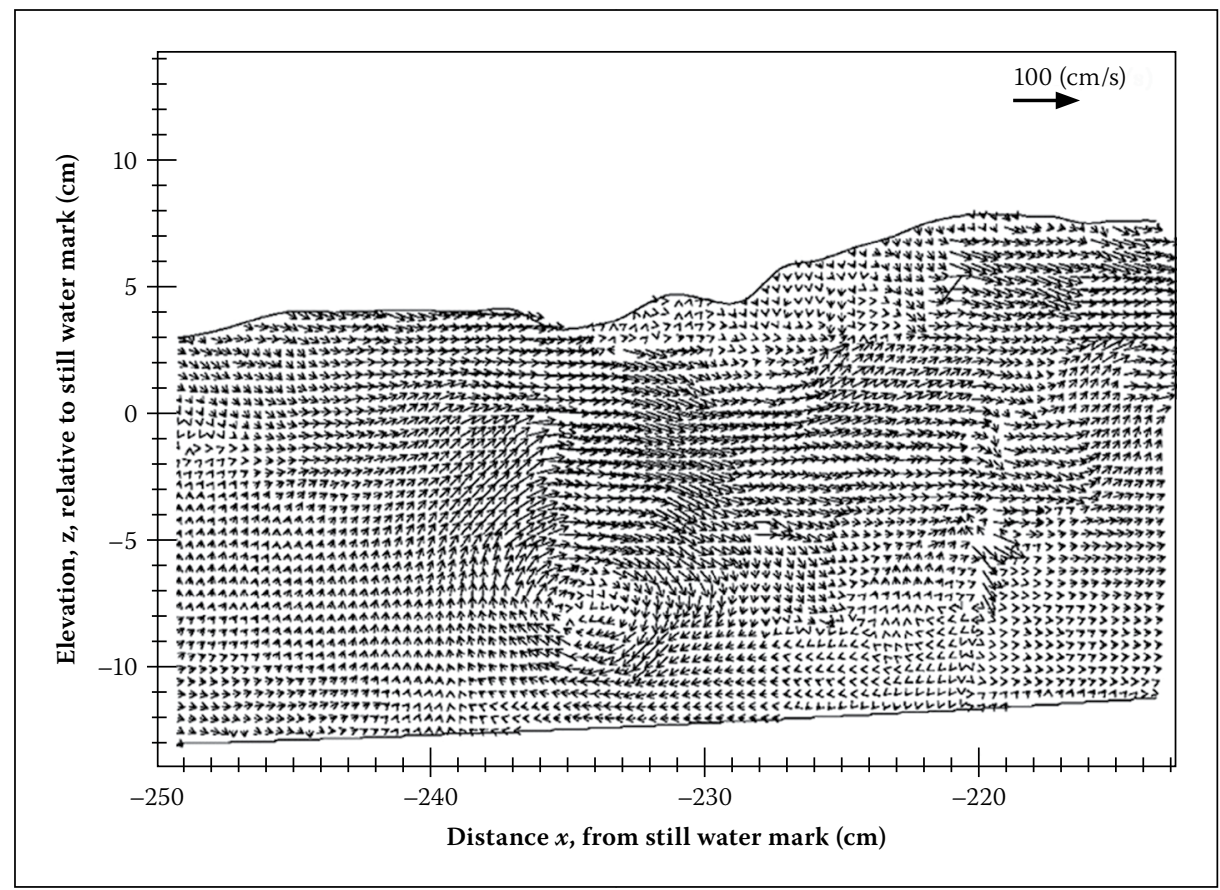

Figure 5 Corresponding instantaneous velocity field for the vorticity field for phase $t / T=0.25$ shown in Figure 4

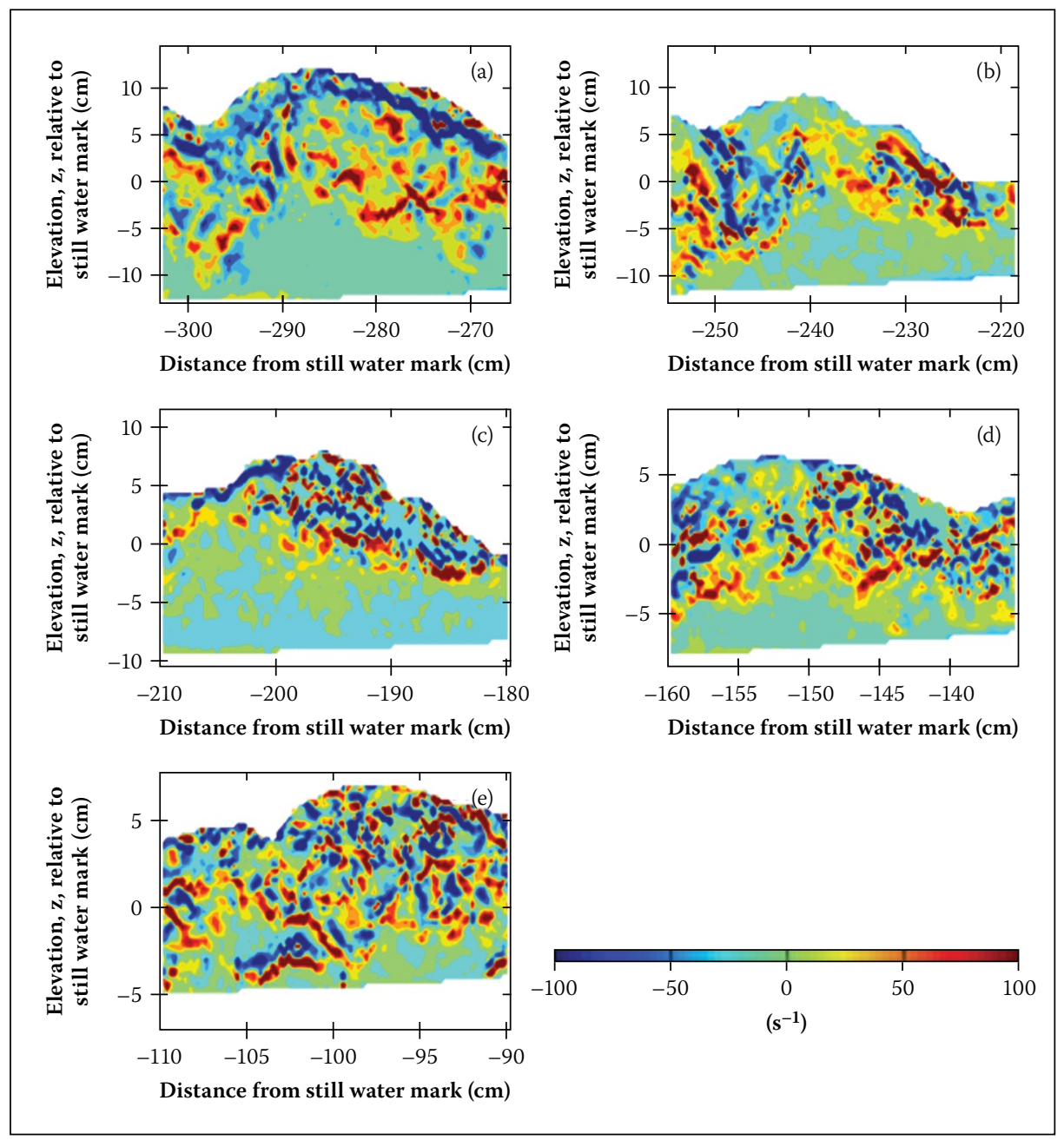

Figure 6 Contour plots of instantaneous vorticity under the wave crests, measured at five different positions along the flume - (a) is furthest from the shore, while (e) is closest; note that there is no time relationship between these plots

frames as the structures travel downstream in the flow, have been estimated. It can be observed that instantaneous vorticity filaments are created in a large range of sizes.
They are estimated to have individual length scales that stretch up to $12 \mathrm{~cm}$ (height of wave used) and a typical mean width of about $2.0 \mathrm{~cm}$. Simulations by Jimenez and
Wray (1998) have shown that the filaments can simultaneously have lengths of the order of the injection scale and a core diameter as small as the Kolmogorov scale. A pair of counter-rotating filaments has an estimated centre separation of about $2.0 \mathrm{~cm}$. Vorticity analysis not only identifies any shearing motion, but also vortex cores present in the flow (Adrian et al 2000). Kevlahan and Farge (1997) noted that most of the time filaments formed during a vortex merger tend to be distributed as a sequence of approximately circular rings around the coherent vortex. In the contour plot of phase 0.20 , a large positive vorticity structure of about $60 \mathrm{~s}^{-1}$ vorticity magnitude is observed, which is centred around $(x, z)=(-250,-7) \mathrm{cm}$. Results show this structure to have developed into a vortex ring that has evolved and moved to position $(x, z)=(-240,-7) \mathrm{cm}$ in the plot of phase 0.25 . Figure 5 shows the instantaneous velocity field that gave rise to vorticity contours presented in the last panel of Figure 4 . The vortex ring is clearly visible in the velocity field.

The wave was divided into 20 phases and the period of wave used is $2.5 \mathrm{~s}$. The time interval between two consecutive phases is $0.125 \mathrm{~s}$. Thus the ring structure has moved about $10 \mathrm{~cm}$ in $0.125 \mathrm{~s}$. This implies that the ring propagates at about $80 \mathrm{~cm} / \mathrm{s}$ towards the shore, which is about $0.74 c$, where $c=108 \mathrm{~cm} / \mathrm{s}$ is the wave phase velocity. This is in agreement with Kimmoun and Branger (2007), who observed that vorticity filaments propagated obliquely towards the bottom, moving more slowly than the wave crest. Miller (1976) traced vortices and noted that they travelled more slowly than the wave velocity, and drifted downwards while expanding.

Figure 6 shows contours of instantaneous vorticity under the wave crests, measured at five different cross-shore positions along the flume. The Figure 6(e) measurements were captured at a point closest to the shore, with its centre only about $1.0 \mathrm{~m}$ from the shore, while the panel in Figure 6(a) is in the deepest water and furthest from the shore, just shoreward of the break point. The flume bed is located at different positions in the panels. While the size of vorticity filaments is observed to decrease towards the shore, there is a general increase in the intensity. Stretching a filament along its axis will make it rotate faster and decrease its width (or diameter in three-dimensions) in order to maintain its angular momentum constant. A well-known example in fluid mechanics is the bath tub vortex that becomes smaller and rotates faster as it goes from the fluid surface to the exit (Petitjeans 2003). Both negative and positive vorticity filaments of magnitude 
up to $100 \mathrm{~s}^{-1}$ can be observed impinging the flume bed as flow progresses from deep water to shallow water. Such high-vorticity filaments are responsible for lifting sediments from the bed and transporting them.

\section{Mean flow vorticity $\left\langle w_{y}\right\rangle$}

Figure 7 shows contour plots of the mean flow vorticity for the six phases of interest. The plots show that the mean flow is characterised by a single, strong, positive vorticity filament located below the crest, at the shear boundary layer. The shear layer arises from the fast-moving mixture of crest water/ bubbles and the opposing slow-moving water near the trough. Mean flow vorticity was obtained by averaging over 100 instantaneous vorticity fields at a particular phase to give the ensemble-averaged vorticity. Mean vorticity distribution shows moderate spatial concentration gradients, in contrast to the patchy instantaneous vorticity. During the early phases of the flow, there is a concentrated region of positive vorticity, about $5 \mathrm{~cm}$ thick near the breaker shear layer. This positive vorticity was due to the shear generated by the high-speed uprush flow overlaying a slower receding downwash layer of fluid (Misra et al 2005). Co-rotating instantaneous filaments observed earlier coalesce to become a larger and stronger single filament. Kevlahan and Farge (1997) observed that strong shearing associated with the vortex mergers produces intense filaments of vorticity. Thus mean flow organises with the formation of a large coherent positive vorticity filament near the shear boundary layer.

Even though the instantaneous flow between the shear layer and the free surface is very turbulent (Figure 4), the observed mean vorticity field is rather weak, except within the shear layer. This is consistent with observations by Lin and Rockwell (1994). Averaging instantaneous vorticity makes small-scale vorticity filaments vanish, so contours of the mean flow vorticity only show the development of large-scale structures within and around the shear layer, which develops and moves downstream. The fluid beneath the elevation of $z=-5 \mathrm{~cm}$ is relatively vorticity-free for the first two phases, but as flow progresses, it is observed that positive vorticity diffuses towards the flume bed. As observed by Sou and Yeh (2011) and presented in Figure 7, the fluid immediately behind the crest (for phases 0.10 to 0.25 ) is characterised by negative vorticity that rides above positive vorticity at elevation centred around $z=-5 \mathrm{~cm}$. As pointed out by Dabiri and Gharib (1997), the negative vorticity seen on the wave, below the surface, and above the positive shear layer above $z=0 \mathrm{~cm}$, indicates the existence of a stagnation point
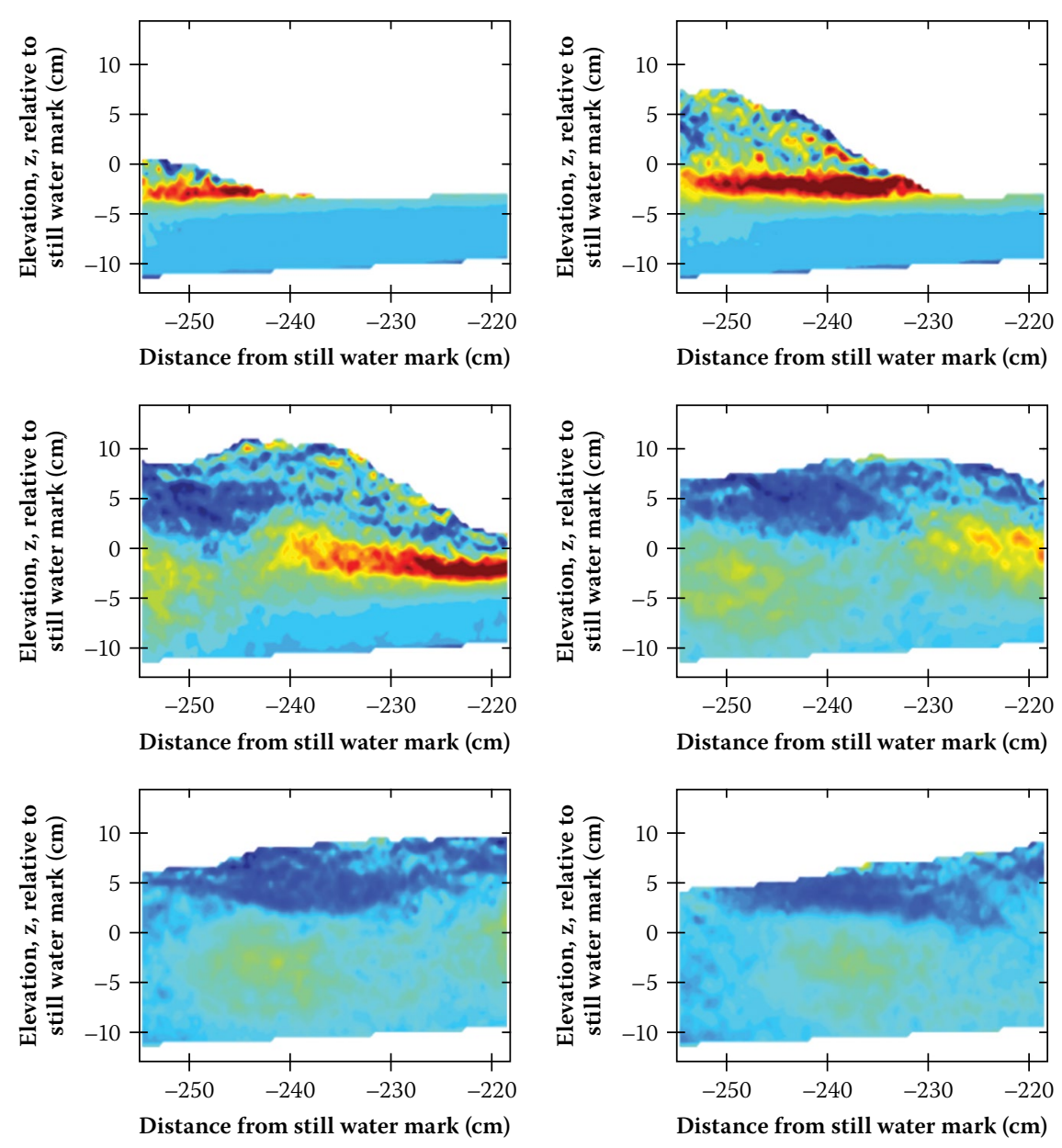

Figure 7 Contour plots showing evolution of the vorticity of the mean flow as flow progressed

at that location. In agreement with observations by Sou and Yeh (2011), the maximum intensity of mean vorticity occurs around the shear layer, and the intensity decreases in magnitude as flow progresses. Thus maximum positive vorticity will remain in the shear layer region between the upper, faster-moving part of the breaking wave and the quiescent region below. Below the trough level mean flow vorticity is about the same order of magnitude as the wave phase speed divided by the local water depth, $c / h=\sqrt{g h} / h$. For the same region, Chang and Liu, (1998; 1999) also found that the vorticity generated by wave breaking was of the same order of magnitude, as the phase speed divided by the local water depth.

Mean vorticity contour plots also reveal the phenomenon of vorticity-shedding at later phases of the flow, in which the tail of the initially strong boundary layer vorticity peels off, weakens and diffuses from the shear boundary layer, reaching the flume bed after the crest has passed. This results in the decay of the vorticity from the neighbourhood of the initially stronger region (Jimenez 2004). The original layer breaks in this way into nearly circular cores. The strong negative vorticity patches near the free surface directly above regions $-255 \mathrm{~cm}<x<-242 \mathrm{~cm}$ for phase 0.10 , and $-242 \mathrm{~cm}<x<-232 \mathrm{~cm}$ for phase 0.15 , may be responsible for the shedding observed. The explanation for the observed decay is that, at later phases, the effects of small-scale mixing due to the strong turbulence in the flow have greatly reduced the presence of patches of vorticity in the flow. It was shown by other researchers (e.g. Stanly et al 2002) that, while the large scales in the flow field adjust slowly to variations in the local mean velocity gradients, the small scales adjust rapidly.

\section{CONCLUSIONS}

Results have been presented of instantaneous and mean vorticity, aimed at determining their evolution as flow progressed. These were estimated from the measured velocity fields using a central difference method.

Results have shown that the spatial distribution of instantaneous vorticity is extremely patchy near the crest, with isolated 
filaments of high positive and negative vorticity that have steep gradients. In-between the patches and below the still water line are large expanses with nearly zero vorticity. Vorticity measurements at different phases at the same point showed that, as flow progressed, there is two-dimensional merging of co-rotating filaments and cancellation of the counterrotating. It was observed that instantaneous vorticity filaments are created in a large range of sizes, peaking at the height of the wave used. Mean vorticity was observed to have completely different characteristics. The principal feature of the phase-averaged vorticity is a strong positive vorticity structure that appears in the flow near the shear boundary layer. A possible reason for this is that vorticity filaments have sizes distributed over several scales present in the flow. Averaging instantaneous vorticity makes small-scale vorticity filaments vanish, so the contours of the mean flow vorticity only show the development of large-scale structures within and around the shear layer, which develops and moves downstream. This occurs near the shear layer, around elevation $z \approx 0$, where the uprush opposes the undertow. At this point a large clockwise-rotating vorticity is formed underneath the wave crest. As the wave crest propagates along the flume, the width of the positive vorticity broadens as it sequentially interacts with downstream vorticity which are remnants of previous breaking waves. This main structure of the wave diffuses rapidly into the interior of the wave after breaking, where molecular diffusion plays a part in stretching the filaments and redistributing the vorticity. Below the trough level, mean flow vorticity is about the same order of magnitude as the wave phase speed divided by the local water depth $c / h=\sqrt{g h} / h$. Peak magnitudes of both instantaneous and mean flow vorticity, generated by wave breaking, are one order of magnitude greater than the phase speed divided by local depth $c / h$.

The results presented here may be useful for modelling two-dimensional turbulent flows. Identification of scaling laws for filaments may also bring some new insights. Statistical properties of the filaments, including filament life time, number of filaments per unit volume, number of high vorticity filaments impinging the bottom and their magnitudes could also be determined from this data, as this information is considered vital in sediment transport studies.

\section{ACKNOWLEDGEMENTS}

The experiments reported here were conducted in the Coastal and Hydraulics Laboratory at the CSIR in Stellenbosch, South Africa. The author would like to acknowledge the technical support received during the experimental runs.

\section{REFERENCES}

Abry, P, Fauve, S, Flandrin, P \& Laroche, C 1994. Analysis of pressure fluctuations in swirling turbulent flows. Journal de Physique II, 4(5): 725-733.

Adrian, R J, Christensen, K T \& Liu Z-C 2000. Analysis and interpretation of turbulent velocity fields. Experiments in Fluids, 29: 275-290.

Aubry, N, Holmes, P, Lumley, J L \& Stone, E 1988. The dynamics of coherent structures in the wall region of a turbulent boundary layer. Journal of Fluid Mechanics, 192: 115-173.

Bakewell, H P, Lumley, J L 1967. Viscous sublayer and adjacent wall region in turbulent pipe flow. Physics of Fluids, 10: 1880-1889.

Belin, F, Maurer, J, Tabeling, P \& Williame, H 1996. Observation of intense filaments in fully developed turbulence. Journal de Physique II (France), 6: 573-583. Brachet, M, Meiron, D, Orszag, S A, Nickel, B, Morf, R \& Frisch, U 1983. Small-scale structure of a Taylor-Green vortex. Journal of Fluid Mechanics, 130: 411-452.

Buhler, O 2000. On the vorticity transport due to dissipating or breaking waves in shallow-water flow. Journal of Fluid Mechanics, 407: 235-263.

Cadot, O, Douady, S \& Couder, Y 1995.

Characterization of the low-pressure filaments in a three-dimensional turbulent shear flow. Physics of Fluids, 7: 630-646.

Chang, K A \& Liu, P L-F 1998. Velocity, acceleration and vorticity under a breaking wave. Physics of Fluids, 10(1): 327-329.

Chang, K A \& Liu, P L-F 1999. Experimental investigation of turbulence generated by breaking waves in water of intermediate depth. Physics of Fluids, 11(11): 3390-3400.

Dabiri, D \& Gharib, M 1997. Experimental investigation of the vorticity generation within a spilling water wave. Journal of Fluid Mechanics, 330: 113-139.

Douady, S, Couder, Y \& Brachet, M-E 1991. Direct observation of the intermittency of intense vorticity filaments in turbulence. Physical Review Letters, 67: 983-986.

Dritschel, D G 1989. The survivability of filaments in two-dimensional fluids at extremely high Reynolds numbers. In: Moffatt, H K \& Tsinober, A (Eds.), Topological Fluid Mechanics, Proceedings of the IUTAM Symposium, Cambridge, UK: Cambridge University Press, p 286.

Dritschel, D G, Haynes, P H, Juckes, M N \& Shepherd, T G 1991. The stability of two-dimensional vorticity filaments under uniform strain. Journal of Fluid Mechanics, 230(1): 647-665.

Elhmaidi, D, Provenzale, A, Lili, T \& Babiano, A 2004. Stability of two-dimensional vorticity filaments. Physics Letters A, 333: 85-90.

Fauve, S, Laroche, C \& Castaing, B 1993. Pressure fluctuations in swirling turbulent flows. Journal de Physique II (France), 3: 271-278.

Galvin, C J 1972. Wave breaking in shallow water. In: Meyer, R E (Ed.), Waves on Beaches and Resultant
Sediment Transport. New York: Academic Press, $413-456$.

Hunt, J C R \& Vassilicos, J C (Eds.) 2000. Turbulence Structure and Vortex Dynamics. Cambridge, UK: Cambridge University Press.

Jimenez, J, Wray, A A, Saffman, P G \& Rogallo, R S 1993. The structure of intense vorticity in isotropic turbulence. Journal of Fluid Mechanics, 255: 65-90.

Jimenez, J \& Wray, A A 1998. On the characteristics of vortex filaments in isotropic turbulence. Journal of Fluid Mechanics, 373: 255-285.

Jimenez, J T 2004. Turbulence and vortex dynamics. Available at: https://www.scribd.com/doc/803204.96/ Javier-Jimenez-Turbulence-and-vortex-dynamics

Keshavarzi, A \& Ball, J 2011. Stochastic nature of flow turbulence and sediment particle entrainment over the ripples at the bed of open channel using image processing technique. In: Bhuiyan, F (Ed.), Sediment Transport - Flow Processes and Morphology, Chapter 3. Rijeka, Croatia: InTech, 69.

Kevlahan, N K-R \& Farge, M 1997. Vorticity filaments in two-dimensional turbulence: Creation, stability and effect. Journal of Fluid Mechanics, 346: 49-76. Kim, W Y, Walker, P G \& Pedersen, E M 1995. Left ventricular blood flow patterns in normal subjects: A quantitative analysis by three-dimensional magnetic resonance velocity mapping. Journal of the American College of Cardiologists, 26, 224-238.

Kimmoun, O \& Branger, H 2007. A particle image velocimetry investigation on laboratory surf-zone breaking waves over a sloping beach. Journal of Fluid Mechanics, 588: 353-397.

Lajeunesse, E, Malverti, L \& Charru, F 2010. Bed load transport in turbulent flow at the grain scale. Experiments and modeling. Journal of Geophysical Research, 115: F04001.

Lee, S J \& Lee, S H 2001. Flow field analysis of a turbulent boundary layer over a riblet surface. Experiments in Fluids, 30: 153-160.

Leweke, T, Dizes, S L, Williamson, I \& Charles, H K 2016. Dynamics and instabilities of vortex pairs. Annual Review of Fluid Mechanics, 48: 1-35.

Lien R-C \& Sanford, T B 2000. Spectral characteristics of velocity and vorticity fluxes in an unstratified turbulent boundary layer. Journal of Geophysical Research, 105(C4): 8659-8672.

Lin, J C \& Rockwell, D 1994. Instantaneous structure of a breaking wave. Physics of Fluids, 6: 2877-2879.

Lin, J, Shao, X \& Ni, L 2002. Wavelet analysis of coherent structures in a three-dimensional mixing layer. Acta Mechanica Sinica, 18(1): 42-52.

Long, J W \& Özkan-Haller, H T 2009. Low-frequency characteristics of wave group-forced vortices. Journal of Geophysical Research, 114: C08004.

Longo, S 2008. Vorticity and intermittency within the pre-breaking region of spilling breakers. Coastal Engineering, 56(3): 285-296.

Meunier, P, Le Dizès, S \& Leweke, T 2005. Physics of vortex merging. Comptes Rendus Physique, 6(4-5): 431-450.

Miller, R L 1976. Role of vortices in surf zone prediction: Sedimentation and wave forces. In: Davis, R A \& Ethington, R L (Eds.), Beach and Nearshore 
Sedimentation, Tulsa, OK: Society of Economic Paleontologists and Mineralogists, 92-114.

Misra, S K, Kirby, J T \& Brocchini, M 2005. The turbulent dynamics of quasi-steady spilling breakers - Theory and experiments. Research Report No. CACR-05-08, Newark, NJ: Center for Applied Coastal Research.

Mukaro, R, Govender, K \& Gledhill, H 2011. Velocity flow fields and water level measurements in shoaling and breaking water waves. Proceedings, 7th South African Conference on Computational and Applied Mechanics, 10-13 January, Pretoria.

Mukaro, R \& Govender, K 2013. Experimental study of turbulence and water levels in shoaling and breaking water waves using digital image processing techniques. Journal of the South African Institution of Civil Engineering, 55(2): 59-74.

Nelson, J M, Shreve, R L, McLean, S R \& Drake, T G 1995. Role of near-bed turbulence structure in bed load transport and bed form mechanics. Water Resources Research, 31: 2071-2086.
Nielsen, P 1984. Field measurements of time-averaged suspended sediment concentration under waves. Coastal Engineering, 8: 51-72.

Petitjeans, P 2003. Stretching of a vortical structure: Filaments of vorticity. Europhysics News, 34(1): 20-23. Raffel, M, Willert, C E, Wereley, S \& Kompenhans, J 1998. Particle Image Velocimetry. A Practical Guide, 2nd ed. Berlin: Springer, 189.

Ryu, Y, Chang, K A \& Mercier, R 2007. Runup and green water velocities due to breaking wave impinging and overtopping. Experiments in Fluids, 43(4): 555-567.

Siggia, E D 1981. Numerical study of small-scale intermittency in three-dimensional turbulence. Journal of Fluid Mechanics, 107: 375-406.

Sou, I M \& Yeh, H 2011. Laboratory study of the crossshore flow structure in the surf and swash zones. Journal of Geophysical Research, 116: C03002.

Stanly, A S, Sarkar, S \& Mellado, J P 2002. A study of the flow-field evolution and mixing in a planar turbulent jet using direct numerical simulation. Journal of Fluid Mechanics, 450: 377-407.
Ting, F C K 2006. Large-scale turbulence under a solitary wave. Coastal Engineering, 53: 441-462.

Ting, F C K 2008. Large-scale turbulence under a solitary wave. Part 2. Forms and evolution of coherent structures. Coastal Engineering, 55: 522-536.

Voulgaris, G \& Collins, M B 2000. Sediment resuspension on beaches: Response to breaking waves. Marine Geology, 167: 167-187.

Wang, L 2012. Structures of the vorticity tube segment in turbulence. Physics of Fluids, 24: 045101.

Williams, J J 1990. Video observations of marine gravel transport. Geo-Marine Letters, 10: 157-164.

Wu, J Z, Ma, H Y \& Zhou, M D 2006. Vorticity and Vortex Dynamics. Berlin-Heidelberg, Germany: Springer.

Zhang, D \& Sunamura, T 1990. Conditions for the occurrence of vortices induced by breaking waves. Coastal Engineering, 33: 145-155.

Zhang, D P 1994. Wave Flume Experiments on the Formation of Longshore Bars Produced by Breaking Waves. Science Report 15, Institute of Geoscience, University Tsukuba, Japan, 47-105. 